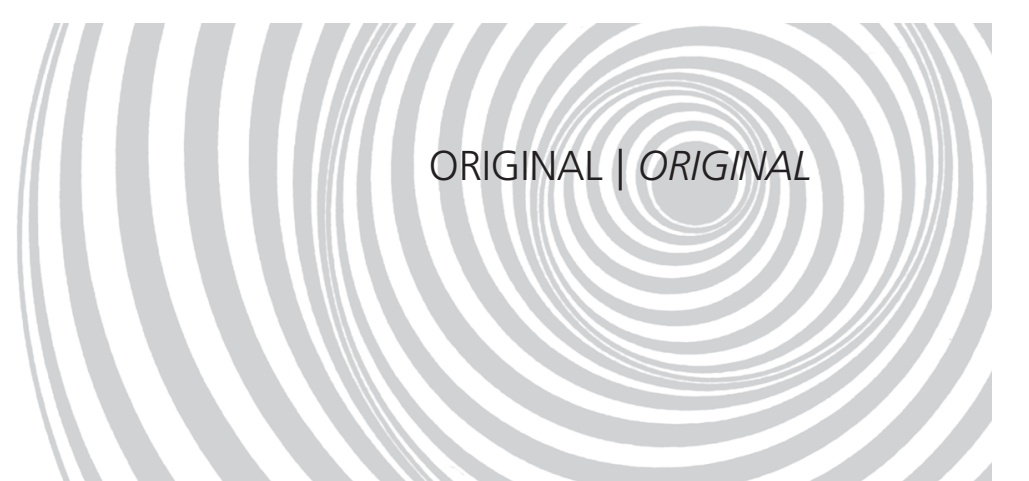

\title{
The three factor eating questionnaire - R21: tradução para o português e aplicação em mulheres brasileiras ${ }^{1}$
}

\author{
The three factor eating questionnaire - R21: \\ translation and administration
}

to Brazilian women

Lara Cristiane NATACCI²

Mario FERREIRA JÚNIOR²

RE S U M O

\section{Objetivo}

Analisar e discutir a relação dos comportamentos de restrição cognitiva, alimentação emocional e descontrole alimentar entre si e com os parâmetros antropométricos: índice de massa corporal e circunferência abdominal.

\section{Métodos}

Tradução para o português e aplicação do The Three Factor Eating Questionnaire - versão reduzida de 21 itens, com subsequente comparação aos parâmetros antropométricos de 125 mulheres trabalhadoras do Instituto Central do Hospital das Clínicas da Faculdade de Medicina da Universidade de São Paulo, escolhidas casualmente em um grupo de 800 interessados em orientação nutricional.

\section{Resultados}

Encontraram-se associações entre a alimentação emocional e o descontrole alimentar, além de associações entre alimentação emocional e descontrole alimentar, índice de massa corporal e circunferência abdominal.

\section{Conclusão}

O The Three Factor Eating Questionnaire - versão reduzida de 21 itens mostrou-se um instrumento adequado para identificar os comportamentos de restrição cognitiva, alimentação emocional e descontrole alimentar, padrões de comportamentos cuja análise pode servir como ponto de partida para a adoção de estratégias de abordagem de orientação nutricional em programas de controle de peso.

Termos de indexação: Comportamento alimentar. Hiperfagia. Estado nutricional.

\footnotetext{
1 Artigo elaborado a partir da dissertação de L.C. NATACCI, intitulada "The Three Factor Eating Questionnaire - R21 (TFEQ-R21): tradução, aplicabilidade, comparação a um questionário semi-quantitativo de frequência de consumo alimentar e a parâmetros antropométricos". Universidade de São Paulo; 2009.

2 Universidade de São Paulo, Hospital das Clínicas, Faculdade de Medicina, Centro de Ensino e Pesquisa em Promoção da Saúde, Serviço de Clínica Geral. Av. Dr. Enéas de Carvalho Aguiar, 155, Prédio dos Ambulatórios, $4^{\circ}$ andar, Bloco C, Cerqueira César, 05403-900, São Paulo, SP, Brasil. Correspondência para/Correspondence to: M. FERREIRA JÚNIOR. E-mails: <mariofj@uol.com.br>; <mario@abps.org.br>.
} 
384 | L.C. NATACCI \& M. FERREIRA JÚNIOR

\section{A B S T R A C T}

\section{Objective}

This study analyzed and discussed how cognitive restraint, emotional eating and bingeing behaviors interrelate and relate with the anthropometric parameters BMI and waist circumference.

\section{Methods}

The short version of The Three Factor Eating Questionnaire consisting of 21 items was translated into Portuguese, administered to 125 female employees from the Central Unit of the Universidade de São Paulo School of Medicine Clinics Hospital and compared with the anthropometric data of these women who had been casually selected from a group of 800 individuals interested in nutrition counseling.

\section{Results}

Emotional eating was found to be associated with bingeing, body mass index and waist circumference.

\section{Conclusion}

The short version of The Three Factor Eating Questionnaire with 21 items proved to effectively identify cognitive restraint, emotional eating and bingeing behaviors. Analysis of these behavior patterns can be the starting point for the implementation of strategies for approaching nutrition counseling in weight-control programs.

Indexing terms: Feeding behavior. Hyperphogia. Nutritional status.

\section{N T R O D U Ç Ã O}

O tratamento da obesidade e das sobrecargas ponderais moderadas constitui atualmente um objeto de controvérsias, assim como o insucesso dos programas de emagrecimento é relatado há décadas ${ }^{1,2}$. O hábito alimentar sofre influência de fatores fisiológicos, psicológicos, cognitivos, comportamentais e sociais ${ }^{3}$. O aumento de peso observado atualmente na população mundial, fruto da abundância e do fácil acesso aos alimentos nas sociedades modernas, pode causar alterações comportamentais descritas sob o nome de restrição cognitiva, que consiste em uma posição mental adotada pelo indivíduo em relação aos alimentos, com o objetivo de reduzir a ingestão energética ${ }^{2}$. O indivíduo em restrição cognitiva se impõe um conjunto de obrigações e proibições alimentares para manter ou perder peso ${ }^{1}$.

No entanto, o comportamento alimentar restritivo pode frequentemente apresentar um fenômeno paradoxal. Enquanto em circunstâncias normais os indivíduos em restrição cognitiva tendem a limitar a ingestão alimentar quantitativa e qualitativamente, muitos deles, quando expostos a certas situações, tendem ao excesso alimentar. Essas situações incluem: ingestão de alimento de alto valor energético', exposição a um alimento "proibido4", estresse ${ }^{5}$, estímulos emocionais negativos $^{6}$, e agentes farmacológicos que afetam o autocontrole, como bebidas alcoólicas ${ }^{7}$. Esse fenômeno paradoxal é descrito como desinibição, uma vez que a situação experimentada desinibe o autocontrole imposto pelo indivíduo quanto a seu comportamento alimentar ${ }^{1,8}$, e apresenta importantes implicações práticas e clínicas ${ }^{9}$.

Por obedecer a regras externas, em detrimento dos sinais fisiológicos de fome e saciedade na regulação do comportamento alimentar, e por alternar fases de restrição e desinibição, a restrição cognitiva tem sido sugerida por alguns autores como um dos fatores responsáveis pelo insucesso das tentativas de emagrecimento ${ }^{1,8}$.

Diversos são os estudos que tentaram identificar a influência das emoções no consumo alimentar 5,6,10-13. O humor e as emoções podem influenciar a escolha dos alimentos, da mesma forma que o consumo de certos alimentos pode alterar o humor ou o estado emocional. Uma questão a ser investigada é se certas características fisiológicas ou psicológicas podem ser preditivas de uma escolha alimentar menos saudável. Weidner et al. ${ }^{14}$ identificaram estudantes que relataram consumir maior quantidade de alimentos mesmo em períodos de restrição alimentar ou 
dieta. Esse dado vai ao encontro de estudos experimentais que apontam que a restrição alimentar pode ser desinibida em casos de estresse ou emoções negativas, como sentir-se entediado, deprimido, ansioso, triste ou tenso $6,11-13,15$. Em contrapartida, quando as escalas de restrição e de alimentação emocional são analisadas separadamente, esta última constitui um melhor indicador de alimentação induzida pelo estresse. Além disso, os indivíduos que apresentam alimentação emocional parecem ser mais susceptíveis aos efeitos do estresse, podendo apresentar mais distúrbios de humor quando em situações desafiadoras, e tentar obter o conforto através do alimento ${ }^{11 .}$

O descontrole alimentar é caracterizado pela perda do autocontrole e consumo exagerado de alimentos, com ou sem a presença de fome ou necessidade orgânica ${ }^{12}$. Sugere-se que a presença dos alimentos e os estímulos sensoriais aumentam as chances de ingestão e descontrole alimentar, tanto em situações de fome quanto em situações de saciedade, indicando que o consumo alimentar nem sempre depende do estado metabólico ${ }^{13}$. Fatores como as oscilações de humor também podem ser antecedentes de consumo exagerado ${ }^{16}$. Recentemente, tem-se relacionado a monotonia alimentar ao descontrole ${ }^{17}$, embora isso ainda possa ser contestado ${ }^{18}$. A consequência mais provável do descontrole alimentar é o aumento de peso e de gordura corporal ${ }^{12}$.

Diante da crescente prevalência do excesso de peso no Brasil ${ }^{19}$ e da aparente importância dos comportamentos acima citados na sua gênese, mostra-se necessário o acesso a um instrumento de pesquisa capaz de identificar indivíduos que estejam em restrição cognitiva ou apresentem sinais de alimentação emocional e/ou descontrole alimentar. Assim, a partir da mensuração dessas características na pessoa ou população que deseja perder peso, será possível propor novas estratégias terapêuticas, possivelmente mais adequadas, realistas e eficazes, como as que envolvem abordagens cognitivo-comportamentais, visando à mudança do hábito alimentar.

Após diversos testes, aplicações experimentais e repetições do processo de identificação de comportamentos associados ao hábito alimentar, foi apresentado, em 1985, um questionário de 51 itens, o The Three Factor Eating Questionnaire $\left(\right.$ TFEQ ${ }^{20}$, reputado como uma medida segura e válida da restrição cognitiva ${ }^{21}$. Após uma modificação parcial pela qual se reduziu o questionário para 18 perguntas ${ }^{22}$, um novo estudo da validade construtiva do questionário resultou no desenvolvimento de uma nova versão, o TFEQ-R2123, agora com 21 itens. Neste último, a escala de restrição cognitiva aborda 6 itens, e afere a proibição alimentar para influenciar o peso ou a forma corporal; a escala de alimentação emocional possui 6 itens e mede a propensão para comer exageradamente em resposta a estados emocionais negativos, como solidão, ansiedade e depressão; e a escala de descontrole alimentar, com 9 itens, verifica a tendência a perder o controle alimentar na presença da fome ou estímulos externos.

O presente estudo objetivou apresentar a tradução do TFEQ-R21 para o português. Além disso, através da sua aplicação preliminar experimental em mulheres que trabalham no Hospital das Clínicas de São Paulo, pretendeu-se identificar a sua consistência como instrumento de avaliação, bem como a correlação entre os comportamentos de restrição cognitiva, alimentação emocional e descontrole alimentar, entre si, e com parâmetros antropométricos que identifiquem o acúmulo de gordura no corpo.

\section{M É T O D O S}

Todos os métodos abaixo descritos constam do projeto de pesquisa submetido e aprovado pelo Comitê de Ética em Pesquisa (CAPPesq) do Hospital das Clínicas da Faculdade de Medicina da Universidade de São Paulo (HCFMUSP), processo $n^{\circ}$ 803/06.

\section{População e amostra de estudo e medidas antropométricas}

Este estudo foi realizado com indivíduos adultos do sexo feminino, de faixa etária entre 
20 e 60 anos, funcionárias, estagiárias ou trabaIhadoras voluntárias do Instituto Central do Hospital das Clínicas da Faculdade de Medicina da Universidade de São Paulo (HCFMUSP), cujos nomes constavam de um cadastro disponível na instituição, indicando o interesse das mesmas em participar de programas que visassem à orientação nutricional.

Através de informações obtidas junto ao Departamento de Recursos Humanos da instituição, o número total de indivíduos cadastrados era de 800 pessoas, já excluídos os homens, as pessoas menores de 20 e maiores de 60 anos de idade, as gestantes e os portadores de marca-passo. Os homens foram excluídos do estudo por constituírem menos de $5 \%$ do total dos indivíduos cadastrados. Para cálculo de amostra, foi usado o programa Sigmastat 3.1, estimando-se uma prevalência de $20 \%$ de distúrbios comportamentais alimentares entre os interessados em programa de redução de peso, e de 5\% para os não interessados. Com poder desejado de $90 \%$ e nível de significância de 0,05, a amostra mínima necessária, estatisticamente significante, foi calculada em 114 indivíduos. Em seguida, a partir da seleção aleatória de 160 indivíduos cadastrados naquela lista, 125 deles aceitaram participar do estudo e assinaram o Termo de Consentimento Livre e Esclarecido (TCLE). As avaliações ocorreram no período de $1^{\circ}$ de novembro de 2006 a 30 de dezembro de 2007, após contato telefônico inicial para agendamento de entrevista.

Todas as participantes foram acolhidas, individualmente, em uma sala do Centro de Ensino e Pesquisa em Promoção de Saúde (CPS) do Serviço de Clínica Geral do HCFMUSP, designada especificamente para que pudessem preencher os formulários de estudo e serem submetidas às medidas antropométricas. Sem calçados e portando roupas leves, tiveram seu peso (massa) e altura mensurados, respectivamente, com uma balança eletrônica Filizola Personal Line modelo PL 150 e um estadiômetro padrão. O Índice de Massa Corporal (IMC) foi calculado através da fórmula: IMC $=P / A^{2}$, na qual P significa peso (massa) atual do paciente em quilos, e $A^{2}$ significa a altura em metros ao quadrado. O IMC constitui um parâmetro de avaliação nutricional de uso muito difundido na área de saúde pública. A Circunferência Abdominal (CA), que auxilia na estimativa do acúmulo de gordura localizada no abdome, foi mensurada com trena antropométrica da marca Sanny, no ponto médio entre a crista ilíaca e a face externa da última costela.

\section{Processo de tradução do questionário}

A versão reduzida do TFEQ-R21 foi traduzida do idioma inglês para o português por dois tradutores juramentados de língua inglesa, em procedimentos independentes. As traduções obtidas em língua portuguesa foram comparadas pelos autores deste estudo, ambos fluentes em inglês, e as diferenças foram conciliadas numa única versão consensual.

O questionário traduzido foi então objeto de um teste piloto com doze indivíduos, escolhidos aleatoriamente, antes de ser aplicado na amostra maior. O objetivo dessa fase foi identificar e resolver qualquer dificuldade de compreensão que surgisse em função da tradução. O teste consistiu na administração do questionário em português, seguida de entrevistas estruturadas com cada pessoa individualmente. A entrevista estruturada abordou cada pergunta do questionário, a fim de determinar se algum dos itens traduzidos foi difícil de responder, confuso ou difícil de entender, e se o entrevistado formularia a pergunta de forma diferente.

Após pequenos ajustes, a versão em português foi então retraduzida (back translation) para a língua inglesa por dois diferentes tradutores juramentados, também em procedimentos independentes. As traduções obtidas foram analisadas pelos autores deste estudo, conciliadas em um único texto e o resultado final comparado ao questionário original. 
Depois de terminado todo o processo de tradução e adaptação, um relatório foi escrito em inglês para o revisor Jan Karlsson, do Health Care Center Unit, Suécia, para revisão e aprovação, contendo todas as traduções e retraduções, sendo a versão final claramente identificada, acompanhada de observações importantes do processo de tradução, mencionando todo o procedimento adotado para se chegar à versão final em português. O revisor emitiu um parecer autorizando o uso do questionário.

\section{Aplicação e interpretação do questionário}

A versão em português do TFEQ-R21, aprovada e autorizada pelos autores estrangeiros do questionário, foi então aplicada às 125 pessoas, durante reunião individual de aproximadamente 30 minutos, que consistiu de três etapas: leitura, análise e assinatura do TCLE e do TFEQ-R21 pelo avaliado; e mensuração antropométrica conduzida pela nutricionista.

Para determinar os graus de restrição cognitiva, alimentação emocional e descontrole alimentar, foram utilizadas as instruções de classificação fornecidas pela equipe que desenvolveu o questionário. Foi utilizado um formato de resposta de 4 pontos para os itens de 1 a 20, e uma escala de classificação numérica de 8 pontos para a questão 21. A média de cada uma das variáveis de comportamento foi calculada e transformada em uma escala de 0 a 100 pontos.

\section{Análise estatística e de consistência do questionário traduzido}

Um teste de confiabilidade da consistência interna do questionário foi realizado calculando-se o coeficiente alfa de Cronbach para medir o desempenho do instrumento na amostra estudada, conforme descrito na literatura24.

Foram estudadas as associações entre os escores das variáveis comportamentais (restrição cognitiva, alimentação emocional e descontrole alimentar) obtidas na análise do TFEQ-R21, relacionadas entre si e com os indicadores antropométricos IMC e CA, através de modelos de regressão linear e cálculo do índice de correlação de Pearson por meio dos programas de estatística Minitab 15.0 e SPSS 12.0. O valor limite de 0,05 foi adotado para determinar o nível de significância dos resultados dos testes estatísticos aplicados.

\section{RESULTADOS}

No Anexo apresenta-se a versão traduzida final, em português, autorizada pelos autores do TFEQ-R21 para uso no Brasil.

Na Tabela 1 constam as estatísticas descritivas da idade e variáveis antropométricas da amostra estudada e dos escores encontrados para cada um dos comportamentos: Restrição Cognitiva (RC), Alimentação Emocional (AE) e Descontrole Alimentar (DA).

Tabela 1. Caracterização da amostra estudada de acordo com a idade, dados antropométricos e escores atribuídos no questionário aos domínios dos comportamentos associados ao hábito alimentar (n=125). São Paulo (SP), 2007.

\begin{tabular}{|c|c|c|c|c|c|}
\hline \multirow{2}{*}{ Variável } & \multirow{2}{*}{ Média } & \multirow{2}{*}{ Desvio-Padrão } & \multirow{2}{*}{ Coeficiente de variação (\%) } & \multicolumn{2}{|c|}{ Valor } \\
\hline & & & & Mínimo & Máximo \\
\hline Idade (anos) & 39,1 & 9,5 & 24,3 & 20,0 & 60,0 \\
\hline Índice de massa corporal (kg/m²) & 27,7 & 5,6 & 20,5 & 18,6 & 47,6 \\
\hline Circunferência abdominal (cm) & 89,1 & 12,2 & 13,7 & 63,0 & 132,0 \\
\hline Descontrole alimentar (0 a 100) & 36,6 & 20,0 & 54,7 & 0,0 & 88,9 \\
\hline Restrição cognitiva (0 a 100) & 48,2 & 19,2 & 39,9 & 0,0 & 83,3 \\
\hline Alimentação emocional (0 a 100) & 39,4 & 28,6 & 72,7 & 0,0 & 100,0 \\
\hline
\end{tabular}


Segundo a análise aplicada à versão definitiva em português do TFEQ-R21, o valor de coeficiente alfa de Cronbach encontrado foi de 0,85. A consistência interna de um instrumento de uso psicométrico será tão melhor quanto mais o coeficiente alfa se aproximar da unidade, portanto, o resultado acima indica boa adequação do instrumento traduzido para os seus propósitos.

Com o objetivo de verificar, preliminarmente, a associação entre as variáveis comportamentais RC, AE e DA, foram aplicados modelos de regressões lineares entre elas. Na Figura 1 são apresentados os gráficos de dispersão para cada par de variáveis comportamentais, com a respectiva reta de ajuste dos modelos de regressão linear e o correspondente coeficiente de correlação linear de Pearson.

A análise das associações dos comportamentos entre si evidencia uma correlação mais forte entre a alimentação emocional e o descontrole alimentar. Não foram encontradas correlações estatisticamente significantes do comportamento de restrição cognitiva com a alimentação emocional nem com o descontrole alimentar.

Foram também avaliadas as relações entre as variáveis comportamentais e duas variáveis antropométricas: IMC e CA. Apesar de os coeficientes de correlação serem baixos, todas as correlações são estatisticamente significantes, o que indica a existência de alguma associação entre os comportamentos ligados ao hábito alimentar e os indicadores de excesso de peso ou acúmulo de gordura, em especial quando se trata de alimentação emocional (Figura 2 e 3).

\section{I S C U S S Ã O}

A experiência prática da aplicação do questionário traduzido, neste estudo, e a análise psicométrica através do valor do alfa de Cronbach demonstraram uma boa adequação da tradução do questionário para os fins aos quais ele se destina. Em 2004, De Lauzon et al. ${ }^{25}$ procederam à análise psicométrica da versão de 18 itens do
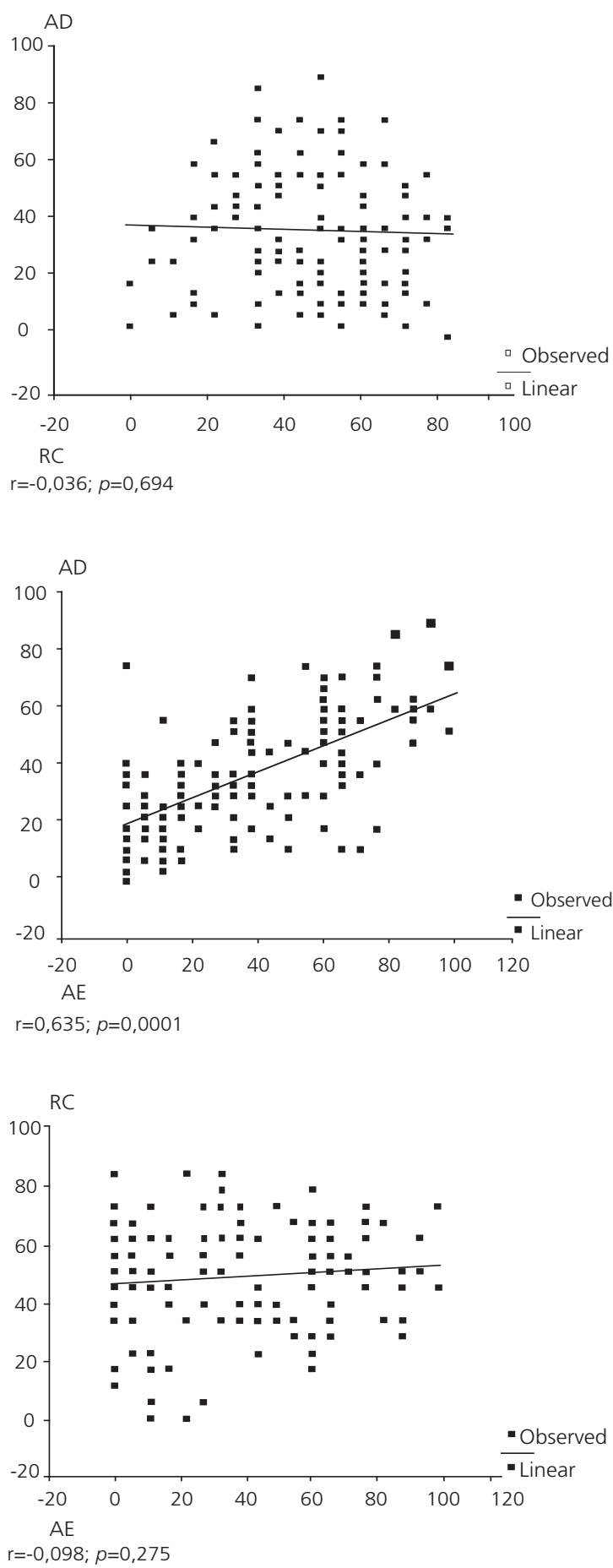

Figura 1. Ilustração do nível de dispersão, retas de regressão linear e índices de correlação de Pearson entre pares dos domínios comportamentais do hábito alimentar - Restrição Cognitiva (RC), Alimentação Emocional (AE) e Descontrole Alimentar (DA) - identificados na amostra avaliada $(n=125)$.

Nota: $r$ : índice de correlação linear de Pearson; $p$ : nível de significância da correlação. 

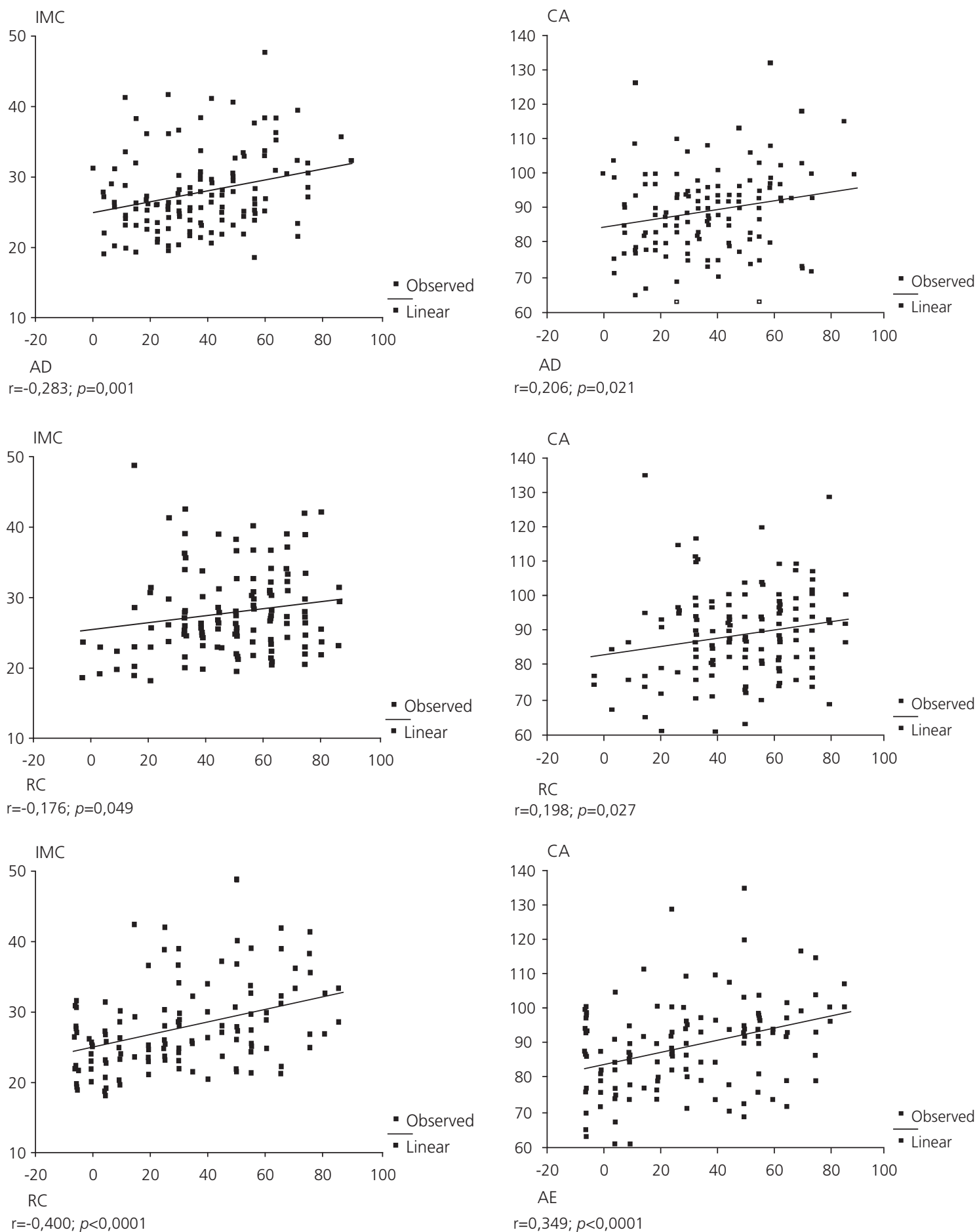

Figura 2. Ilustração do nível de dispersão, retas de regressão linear e índices de correlação de Pearson entre a variável Índice de Massa Corporeal (IMC) e os domínios comportamentais do hábito alimentar - Restrição Cognitiva (RC), Alimentação Emocional (AE) e Descontrole Alimentar (DA) - Identificados na Amostra Avaliada ( $n=125)$.

Nota: r: índice de correlação linear de Pearson; $p$ : nível de significância da correlação.

Figura 3. Ilustração do nível de dispersão, retas de regressão linear e índices de correlação de Pearson entre a variável Circunferência Abdominal (CA) e os domínios comportamentais do hábito alimentar - Restrição Cognitiva (RC), Alimentação Emocional (AE) e Descontrole Alimentar (DA) - identificados na amostra avaliada $(n=125)$.

Nota: $r$ : índice de correlação linear de Pearson; $p$ : nivel de significância da correlação. 
TFEQ e encontraram um resultado igualmente satisfatório. Isso indica que a aplicação do TFEQ-R21 em português tem a capacidade de expressar, com alto grau de confiabilidade, o comportamento dos brasileiros em relação a todos os domínios que ele aborda.

Muito embora o TFEQ-R21 tenha sido originalmente desenvolvido para análise do comportamento alimentar de indivíduos obesos, estudos mais recentes identificaram sua validade também em populações eutróficas ${ }^{25,26}$.

Tendo-se em conta que, na amostra das pessoas estudadas, as distribuições dos valores de IMC e de CA sugeriam maior prevalência do excesso de peso e acúmulo de gordura abdominal (com variação da ordem de $20 \%$ a $25 \%$ ) e que as participantes da pesquisa, trabalhadoras de um grande hospital de São Paulo, faziam parte de um cadastro de pessoas que já haviam expressado o interesse de participar de programas de orientação nutricional, era esperado que a aplicação do questionário revelasse comportamentos disfuncionais ligados a hábitos alimentares.

De fato, a análise dos escores atribuídos aos três domínios de comportamento mostrou que o TFEQ-R21 foi correto na tentativa de identificá-los, e que os resultados estão de acordo com estudos anteriores, que ressaltaram que a alimentação emocional, e não a restrição cognitiva, destaca-se como um dos maiores responsáveis pelo descontrole alimentar e o consumo energético exagerado, resultando em aumento de peso, conforme já demonstrado por Westerhofer ${ }^{27}$.

Porém, ao contrário do que mostraram outros estudos realizados em países do hemisfério norte ${ }^{27,28}$, a restrição cognitiva apesar de presente na amostra estudada, não se correlacionou com os domínios da alimentação emocional e do descontrole alimentar, e associou-se pouco com os indicadores de excesso de peso e acúmulo de gordura. Essa discordância em relação a outros estudos poderia significar um baixo desempenho do questionário na abordagem dos comportamentos; porém, como o domínio de alimentação emocional associou-se fortemente ao descontrole alimentar e outros indicadores, surgem algumas hipóteses: que possivelmente o grupo avaliado neste estudo estivesse sob influência preponderante de fatores desencadeantes da alimentação emocional (por exemplo, estresse no trabalho); que esta possa ser decorrente de aspectos culturais da população brasileira, que se comportaria diferentemente de outras do hemisfério norte; que a característica transversal deste estudo tenha enviesado os resultados, uma vez que ignora variações comportamentais ao longo do tempo.

Uma relação positiva entre a restrição cognitiva e a alimentação emocional foi reportada anteriormente em estudos de coorte, como o conduzido por Lindroos em 199729, e confirmada por Provencher et al. ${ }^{30}$, relatando que a restrição cognitiva pode deixar o indivíduo vulnerável à alimentação emocional e mais reativo à exposição sensorial ou cognitiva ligada ao alimento. Portanto, é possível que o acompanhamento da amostra estudada ao longo do tempo viesse a demonstrar uma alternância entre os comportamentos alimentares avaliados.

Esses achados preliminares, conseguidos com o uso do TFEQ-R21, na versão em português, são interessantes e levantam várias dúvidas em relação aos comportamentos associados aos hábitos alimentares de brasileiros, que podem estar impactando de forma decisiva a prevalência crescente do ganho de peso no país. Ao mesmo tempo, abrem uma gama de possibilidades de abordagens terapêuticas que somam à orientação nutricional clássica, baseada principalmente no conteúdo energético dos alimentos e dietas restritivas, os conhecimentos acumulados e as técnicas da psicologia comportamental no aconselhamento de hábitos alimentares mais saudáveis e eficazes, visando ao controle ou redução do peso e acúmulo de gordura corporal.

\section{O N CLUSÃ O}

Este estudo revelou que a versão em português do TFEQ-R21, autorizada pelos autores para aplicação no Brasil, pode ser um instrumento ade- 
quado para identificar os comportamentos de restrição cognitiva, alimentação emocional e descontrole alimentar, associados ao hábito alimentar.

Revelou também que a alimentação emocional foi o fator melhor relacionado ao descontrole alimentar nas mulheres brasileiras estudadas, assim como aos indicadores de excesso de peso e acúmulo de gordura corporal, e que a restrição cognitiva contribuiu pouco para o agravamento da alimentação descontrolada.

Esses resultados sugerem que, com o uso do TFEQ-R21 em estudos transversais ou longitudinais mais aprofundados, a identificação dos comportamentos associados podem servir de ponto de partida para a adoção de estratégias de abordagem na orientação nutricional, que levem em conta não apenas o conteúdo energético dos alimentos ou a recomendação de dietas restritivas, mas que também valorizem os comportamentos desencadeantes da alimentação inadequada, como por exemplo, estímulos externos e emoções.

\section{COLABORADORES}

L.C. NATACCI responsável por planejamento da pesquisa, trabalhos de campo e redação do artigo. M. FERREIRA JUNIOR responsável pelas orientações gerais, planejamento da pesquisa, análise estatística e redação do artigo.

\section{REFERÊ NCIAS}

1. Herman $C P$, Mack D. Restrained and unrestrained eating. J Pers. 1975; 43(4):647-60.

2. Lowe MR, Timko CA. What a difference a diet makes: towards an understanding of differences between restrained dieters and restrained nondieters. Eat Behav. 2004; 5(3):199-208.

3. Apfeldorfer G, Zermati JP. Cognitive restraint in obesity: history of ideas, clinical description. Presse Med. 2001; 30(32):1575-80.

4. O'Connell C, Larkin K, Mizes JS, Fremouw W. The impact of caloric preloading on attempts at food and eating-related thought suppression in restrained and unrestrained eaters. Int J Eat Disord. 2005; 8(1):42-8.
5. Wallis DJ, Hetherington MM. Stress and eating: the effects of ego-threat and cognitive demand on food intake in restrained and emotional eaters. Appetite. 2004; 43(1):39-46.

6. Heo M, Pietrobelli A, Fontaine KR, Sirey JA, Faith MS. Depressive mood and obesity in US adults: comparison and moderation by sex, age, and race. Int J Obes. 2006; 30(3):513-9.

7. Polivy J, Herman CP. Effects of alcohol on eating behavior: influence of mood and perceived intoxication. J Abnorm Psychol. 1976; 85(6):601-6.

8. Le Barzic M. Le syndrome de restriction cognitive: de la norme au désordre du comportement alimentaire. Diabetes Metab. 2001; 27:512-6.

9. Spencer JA, Fremouw WJ. Binge eating as a function of restraint and weight classification J Abnorm Psychol. 1979; 88(3):262-7.

10. Herman CP, Polivy J. Anxiety, restraint, and eating behavior. J Abnorm Psychol. 1975; 84(6):66-72.

11. Rutters F, Nieuwenhuizen AG, Lemmens SG, Born JM, Westenterp-Plantenga MS. Acute Stress-related changes in eating in the absence of hunger. Obesity. 2008; 17:72-7.

12. Gilhooly CH, Das SK, Golden JK, McCrory MA, Dallal GE, Saltzman E, et al. Food cravings and energy regulation: the characteristics of craved foods and their relationship with eating behaviors and weight change during 6 months of dietary energy restriction. Int J Obes. 2007; 31(12): 1849-58.

13. Lambert KG, Neal T, Noyes J, Parker C, Worrel P. Food-related stimuli increase desire to eat in hungry and satiated human subjects. Curr Psychol Res Rev. 1991; 10(4):297-303.

14. Weidner G, Kohlmann CW, Dotzauer E, Burns LR. The effects of academic stress on health behaviors in young adults. Anxiety Stress Coping. 1996; 9(2): 123-33.

15. Epel E, Lapidus R, McEwen B, Brownell K. Stress may add bite to appetite in women: a laboratory study of stress-induced cortisol and eating behavior. Psychoneuroendocrinology. 2001; 26:37-49.

16. Tiggemann $M$, Kemps $E$. The phenomenology of food cravings: the role of mental imagery. Appetite. 2005; 45(3):305-13.

17. Pelchat ML, Schaefer S. Dietary monotony and food cravings in young and elderly adults. Physiol Behav. 2000; 68(3):353-9.

18. Martin CK, O’Neil PM, Pawlow L. Changes in food cravings during low-calorie and very-low-calorie diets. Obesity. 2006; 14(1):115-21.

19. Instituto Brasileiro de Geografia e Estatística. Pesquisa de orçamentos familiares, 2002-2003. Aná- 
lise da disponibilidade domiciliar de alimentos e do estado nutricional no Brasil [Internet]. Rio de Janeiro: IBGE; 2004. Disponível em: <http://www. ibge.gov.br>.

20. Stunkard AJ, Messick S. The Three Factor Eating Questionnaire to measure dietary restraint, disinhibition and hunger. J Psychosom Res. 1985; 29(1):71-83.

21. Laessle RG, Tuschl RJ, Kotthaus BC, Pirke KM. A comparison of the validity of three scales for the assessment of dietary restraint. J Abnorm Psychol. 1989; 98(4):504-7.

22. Karlsson J, Persson LO, Sjöström L, Sullivan M. Psychometric properties and factor structure of the Three Factor Eating Questionnaire (TFEQ) in obese men and women. Results from the Swedish Obese Subjects (SOS) study. Int J Obesity. 2000; 24(12): 1715-25.

23. Tholin S, Rasmussen F, Tynelius P, Karlsson J. Genetic and environmental influences on eating behaviour: the Swedish young male twins study. Am J Clin Nutr. 2005; 81:564-9.

24. Cronbach LJ, Warrington WG. Time-limit tests: estimating their reliability and degree of speeding 1. Psychometrika. 1951; 16(2):167-88.

25. Lauzon B, Romon M, Deschamps V, Lafay L, Borys JM, Karlsson J, et al. Fleurbaix Laventie Ville Sante Study Group. The Three-Factor Eating Questionnaire-
R18 is able to distinguish among different eating patterns in a general population. J Nutr. 2004; 134(9):2372-80.

26. Hyland ME, Irvine SH, Thacker C, Dann PL, Dennis I. Psychometric analysis of the Stunkard-Messick Eating Questionnaire (SMEQ) and comparison with the Dutch Eating Behavior Questionnaire (DEBQ). Curr Psychol Res Rev. 1989; 8:228-33.

27. Westenhofer J, Broeckmann P, Munch AK, Pudel V. Cognitive control of eating behaviour and the deshinibition effect. Appetite. 1994, 23:27-41.

28. Westenhofer J, Stunkard AJ, Pudel V. Validation of the subscale of the flexible and rigid control dimensions of dietary restraint. Int J Eat Disord. 1999; 26(1):53-64.

29. Lindroos AK, Lissner L, Mathiassen ME, Karlsson J, Sullivan $M$, Bengtsson C, et al. Dietary intake in relation to restrained eating, disinhibition, and hunger in obese and nonobese Swedish women. Obes Res. 1997; 5(3):175-82.

30. Provenches V, Drapeau V, Tremblay A, Després JP, Bouchard C, Lemieux S. Eating behaviours, dietary profile and body composition according to dieting history in men and women of the Quebéc Family Study. Br J Nutr. 2004; 91:977-1004.

Recebido em: 8/6/2009

Versão final reapresentada em: 21/7/2010 Aprovado em: 16/12/2010 


\section{ANEXO}

\section{QUESTIONÁRIO TFEQ-R21 - VERSÃO EM PORTUGUÊS}

Esta seção contém declarações e perguntas sobre hábitos alimentares e sensação de fome.

Leia cuidadosamente cada declaração e responda marcando a alternativa que melhor se aplica a você.

1. Eu deliberadamente consumo pequenas porções para controlar meu peso

$\square$ Totalmente verdade

$\square$ Verdade, na maioria das vezes

$\square$ Falso, na maioria das vezes

$\square$ Totalmente falso

2. Eu começo a comer quando me sinto ansioso.

$\square$ Totalmente verdade

$\square$ Verdade, na maioria das vezes

$\square$ Falso, na maioria das vezes

$\square$ Totalmente falso

3. Às vezes, quando começo a comer, parece-me que não conseguirei parar.

$\square$ Totalmente verdade

$\square$ Verdade, na maioria das vezes

$\square$ Falso, na maioria das vezes

$\square$ Totalmente falso

4. Quando me sinto triste, frequentemente como demais.

$\square$ Totalmente verdade

$\square$ Verdade, na maioria das vezes

$\square$ Falso, na maioria das vezes

$\square$ Totalmente falso

5. Eu não como alguns alimentos porque eles me engordam.

$\square$ Totalmente verdade

$\square$ Verdade, na maioria das vezes

$\square$ Falso, na maioria das vezes

$\square$ Totalmente falso

6. Estar com alguém que está comendo, me dá frequentemente vontade de comer também.

$\square$ Totalmente verdade

$\square$ Verdade, na maioria das vezes

Falso, na maioria das vezes

$\square$ Totalmente falso

7. Quando me sinto tenso ou estressado, frequentemente sinto que preciso comer.

$\square$ Totalmente verdade

$\square$ Verdade, na maioria das vezes

$\square$ Falso, na maioria das vezes

$\square$ Totalmente falso

8. Frequentemente sinto tanta fome que meu estômago parece um poço sem fundo.

$\square$ Totalmente verdade

$\square$ Verdade, na maioria das vezes

$\square$ Falso, na maioria das vezes

$\square$ Totalmente falso

9. Eu sempre estou com tanta fome, que me é difícil parar de comer antes de terminar toda a comida que está no prato.

$\square$ Totalmente verdade

$\square$ Verdade, na maioria das vezes

$\square$ Falso, na maioria das vezes

$\square$ Totalmente falso

10. Quando me sinto solitário (a), me consolo comendo.

$\square$ Totalmente verdade

$\square$ Verdade, na maioria das vezes

$\square$ Falso, na maioria das vezes

$\square$ Totalmente falso 
394 | L.C. NATACCI \& M. FERREIRA JÚNIOR

11. Eu conscientemente me controlo nas refeições para evitar ganhar peso.

$\square$ Totalmente verdade

$\square$ Verdade, na maioria das vezes

$\square$ Falso, na maioria das vezes

$\square$ Totalmente falso

12. Quando sinto o cheiro de um bife grelhado ou de um pedaço suculento de carne, acho muito difícil evitar de comer, mesmo que eu tenha terminado de comer há muito pouco tempo.

$\square$ Totalmente verdade

$\square$ Verdade, na maioria das vezes

$\square$ Falso, na maioria das vezes

$\square$ Totalmente falso

13. Estou sempre com fome o bastante para comer a qualquer hora.

$\square$ Totalmente verdade

$\square$ Verdade, na maioria das vezes

$\square$ Falso, na maioria das vezes

$\square$ Totalmente falso

14. Se eu me sinto nervoso(a), tento me acalmar comendo.

$\square$ Totalmente verdade

$\square$ Verdade, na maioria das vezes

$\square$ Falso, na maioria das vezes

$\square$ Totalmente falso

15. Quando vejo algo que me parece muito delicioso, eu frequentemente fico com tanta fome que tenho que comer imediatamente.

$\square$ Totalmente verdade

$\square$ Verdade, na maioria das vezes

$\square$ Falso, na maioria das vezes

$\square$ Totalmente falso

16. Quando me sinto depressivo(a), eu quero comer.

$\square$ Totalmente verdade

$\square$ Verdade, na maioria das vezes

$\square$ Falso, na maioria das vezes

$\square$ Totalmente falso

17. O quanto frequentemente você evita "estocar" (ou se aprovisionar de) comidas tentadoras?

$\square$ Quase nunca

$\square$ Raramente

$\square$ Freqüentemente

$\square$ Quase sempre

18. O quanto você estaria disposto(a) a fazer um esforço para comer menos do que deseja?

$\square$ Não estou disposto(a)

$\square$ Estou um pouco disposto(a)

$\square$ Estou relativamente bem disposto(a)

$\square$ Estou muito disposto(a)

19. Você comete excessos alimentares, mesmo quando não está com fome?

$\square$ Nunca

$\square$ Raramente

$\square$ Às vezes

$\square$ Pelo menos 1 vez por semana

20. Com qual frequência você fica com fome?

$\square$ Somente nos horários das refeições

$\square$ Às vezes entre as refeições

$\square$ Frequentemente entre as refeições

$\square$ Quase sempre

21. Em uma escala de 1 a 8, onde 1 significa nenhuma restrição alimentar, e 8 significa restrição total, qual número você daria para si mesmo?

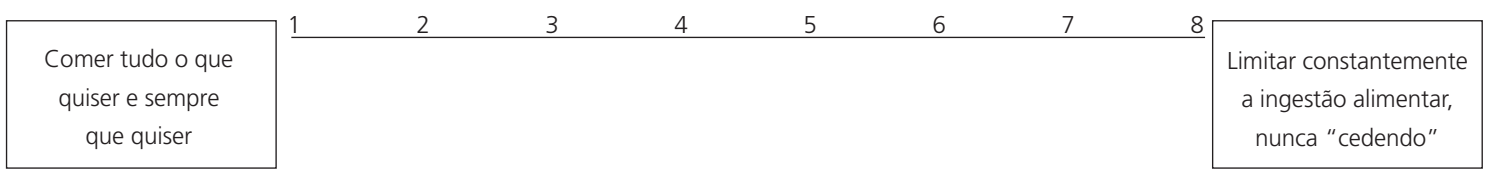

